\title{
An innovative system for uncoupled bending/torsion tests by tri-axis shaker: numerical simulations and experimental results
}

\author{
Davide Zanellati ${ }^{1}$, Denis Benasciutti ${ }^{1}$, and Roberto Tovo ${ }^{1}$ \\ ${ }^{1}$ Department of Engineering, University of Ferrara, via Saragat 1, 44122, Ferrara, Italy
}

\begin{abstract}
This work describes a new testing system for applying a coupled/uncoupled bendingtorsion loading in vibratory tests by a tri-axis shaker. The system is composed of a cylindrical specimen with eccentric tip masses, excited by horizontal and/or vertical base accelerations. The specimen tip is constrained by a lateral thin and flexible plate which impedes any bending when the specimen is excited horizontally, but which permits the specimen torsional rotation. This layout then allows torsional and bending deformations to be produced and controlled independently, when vertical and horizontal base accelerations are applied simultaneously. A finite element model is first used to estimate the system dynamic response and the stresses in the notched specimen section. The model is then validated through experimental tests under harmonic base accelerations. The strains at clamping system are also monitored to indirectly estimate the bending and torsion moment in the specimen. Comparison of numerical and experimental results showed a close correlation and proved that bending-torsion loading are truly uncoupled. Preliminary fatigue tests with harmonic bending loading (vertical base excitation) are finally compared to the constant amplitude S-N curve, showing a quite satisfactory agreement.
\end{abstract}

\section{Introduction}

Mechanical systems and structures are often subjected to vibratory excitations, which generally induce a local multiaxial random stress. In the structural durability assessment, besides the use of theoretical and numerical methods implementing uniaxial/multiaxial criteria (often defined in the frequency-domain [1-5]), it is customary to use experimental tests to directly estimate the material strength against multiaxial loading. The interest is often restricted to bending-torsion random loading, as they are very common in most engineering applications.

Laboratory experiments with multiaxial loading are normally performed in two different ways: by servohydraulic machines that impose a force and/or torque (testing frequencies are relatively low), or by shaker tables that apply a base vibratory excitation (thus allowing for higher testing frequencies).

Over the years, a number of testing methodologies that differ in terms of testing machines, specimen geometry and type of excitation, have been proposed [610]. While servo-hydraulic machines normally adopt cylindrical specimens, vibratory tests by shakers tables make use of a wider set of specimen configurations, ranging from simple (plate, cylindrical) to more elaborated layouts (Y-geometry), as already commented in [11]. In vibratory testing, the specimen is fixed to the shaker table and excited by a base acceleration.

For what concerns, in particular, tests with a biaxial bending-torsion loading, both testing methodologies offer advantages and disadvantages. While servohydraulic machines permit a great flexibility in controlling both the intensity and phase shift of bending and/or torsion loading, shaker tables seem to have greater limitations. For example, in most of vibratory test configurations that use a uniaxial shaker, only a uniaxial stress can be obtained (bending loading). A bendingtorsion loading can only be obtained (at a greater cost) by coupling two uniaxial shakers controlled independently [8].

A simple way to obtain a bending-torsion loading by a uniaxial shaker is the use of a cantilever specimen with eccentric mass at the free extremity, as suggested in [10]. In fact, the eccentricity of the tip masses induces a torsion loading (combined to bending), even when the specimen is excited by uniaxial vertical acceleration. The intensity of bending loading is controlled by the mass weight, the intensity of torsion loading is determined by the degree of eccentricity. While a zero eccentricity would give only bending loading (without torsion), the opposite would not be possible (torsion loading is always coupled to bending). Therefore, this configuration cannot produce a torsion loading alone. The use of a tri-axis shaker, which can apply three independent excitations

*Corresponding author: davide.zanellati@unife.it 
along three orthogonal directions, seems to open the possibility to overcome this limitation. Inspired by the layout proposed in [10], a new testing system has been proposed, which allowed torsion and bending loading to be controlled independently in a vibratory test with a triaxial shaker [11]. The purpose of this work is to describe this innovative testing system, to present results from a finite element model and to validate them against experiments, and finally to show preliminary fatigue test results under a harmonic bending loading.

\section{Layout of innovative testing system:}

The innovative testing system layout (Fig.1) is composed of a T-clamping system, a lateral thin plate and a Unotched cylindrical specimen in S355JR steel. The diameters of gross and net cross sections are 20 and 8 $\mathrm{mm}$, respectively. The notch radius of $2 \mathrm{~mm}$ gives a stress concentration factor $K_{\mathrm{t}, \mathrm{b}}=1.45$ for bending and $K_{\mathrm{t}, \mathrm{t}}=1.27$ for torsion [12]. A cantilever beam with two equal tip masses is mounted at the specimen free extremity. The overall weight of the system is $4.65 \mathrm{~kg}$.

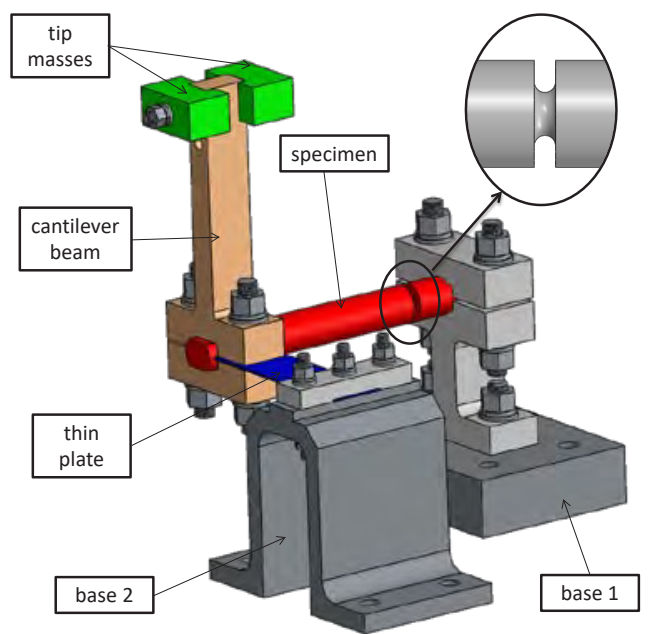

Fig. 1. Layout of testing system. (Reprinted from [11], with permission from Elsevier)

Both the specimen extremity and the base of the cantilever beam are constrained by a thin plate. The purpose of the plate is to prevent any horizontal displacement. It is also very thin, and thus flexible enough, to allow any torsional rotation of the specimen. Therefore, a horizontal base excitation could cause only a torsional loading induced by the eccentric tip masses, whereas the horizontal bending is completely impeded by the thin plate. A vertical excitation, instead, could induce only a bending loading on the vertical plane, without any torsional rotation.

In order to verify this system behaviour, a finite element model was first developed.

\section{Finite element model}

Through a modal and harmonic analysis, the finite element model aims to prove that the innovative testing layout in Fig. 1 allows bending and torsion loading to be fully uncoupled under a horizontal and vertical base acceleration.

The numerical model assumes a isotropic linear elastic material, with density $8027 \mathrm{~kg} / \mathrm{m}^{3}$, Young's modulus $200 \mathrm{GPa}$ and Poisson's ratio 0.3, typical values of a S355JR steel.

A structured mesh was used everywhere, except on the base 1 due to its complex geometry (Fig. 2). The average element size in the notch is less than $0.5 \mathrm{~mm}$, instead it is $1 \mathrm{~mm}$ elsewhere. A 8-node solid finite element, with three degrees of freedom for each node, was adopted. The nodes lying on the bottom surfaces of the clamped base (base 1 and base 2 in Fig. 1) were fully constrained.

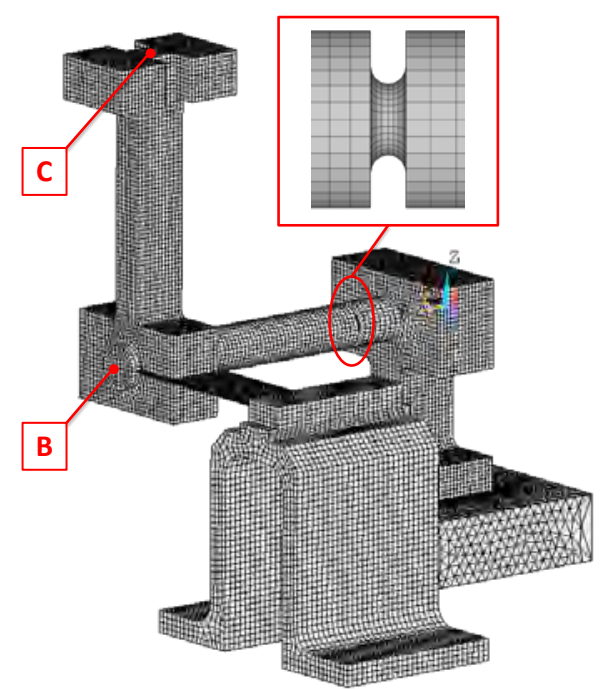

Fig. 2. Finite element model. (Reprinted from [11], with permission from Elsevier).

A modal analysis was performed to identify the first two resonance frequencies of the system; the first bending resonance is at $78.7 \mathrm{~Hz}$, the first torsion resonance occurs at $110 \mathrm{~Hz}$. The corresponding modal shapes are displayed in Fig. 3. The other resonances at higher frequencies were not considered, as they refer to modal shapes of the whole system (not only the specimen).
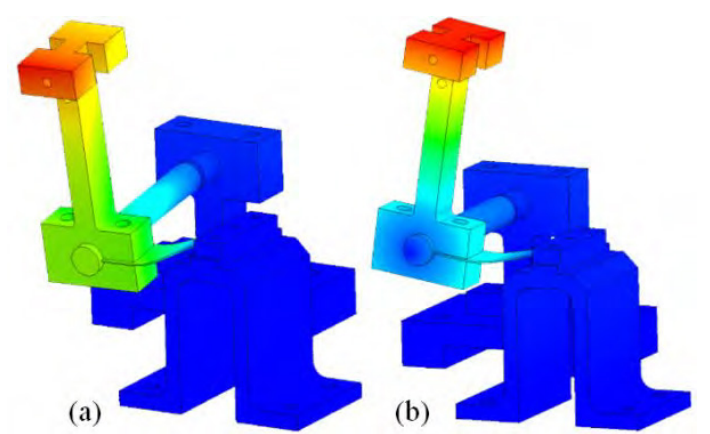

Fig. 3. Modal shape in (a) bending and (b) in torsion. (Reprinted from [11], with permission from Elsevier).

Two harmonic analyses were also performed to determine the frequency response function (FRF) of the system under a vertical or horizontal harmonic acceleration, respectively, which induce a pure bending or torsion loading in the specimen. A harmonic 
acceleration with $1 g$ of amplitude and frequency range from 20 to $150 \mathrm{~Hz}$ was applied as an enforced motion at the base. The acceleration response (vertical and horizontal) was determined at points B and C (Fig. 2).

In the first harmonic analysis, a vertical acceleration was applied to produce a pure bending loading and a corresponding normal stress in the notched section of the specimen. Fig. 4 shows, with a solid line, the FRF in terms of vertical acceleration at the specimen extremity (point B in Fig. 2).

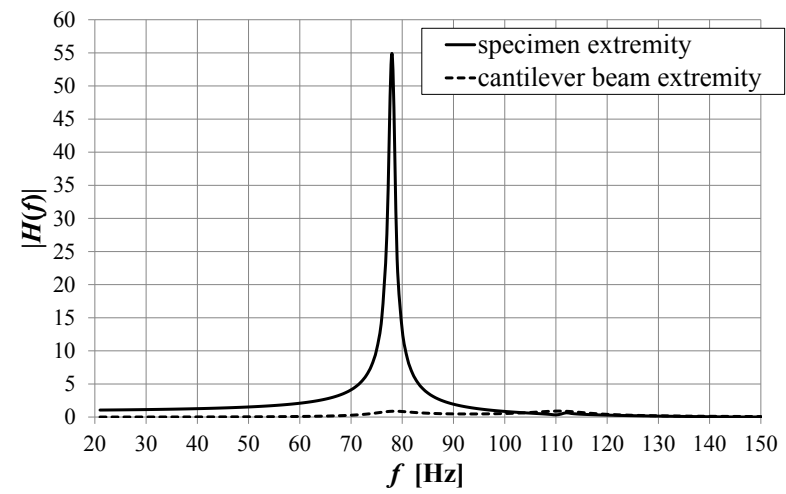

Fig. 4. Amplitude FRF $|\mathrm{H}(\mathrm{f})|$ under a vertical acceleration.

The typical resonant behaviour is observed. At low frequencies, the system response is close to one (quasistatic behaviour), whereas when approaching the first resonance frequency, as expected, the system response is greatly magnified. At frequencies higher than resonance, the FRF decreases. Fig. 4 also displays, with a dashed line, the FRF in terms of horizontal acceleration of the cantilever extremity (point $\mathrm{C}$ in Fig. 2), which is an indirect measure of the torsional deformation. In this case, the FRF is close to zero at low frequencies and exhibits a little amplification at the two resonances. At frequencies higher than resonance, the system response returns to zero. The two small peaks in Fig. 4 can be explained by the action of the thin plate attached to the specimen: when the specimen bends vertically, the plate cannot elongate and therefore it restraints the specimen, which is then forced to slightly rotate and to produce a corresponding horizontal displacement of point $\mathrm{C}$.

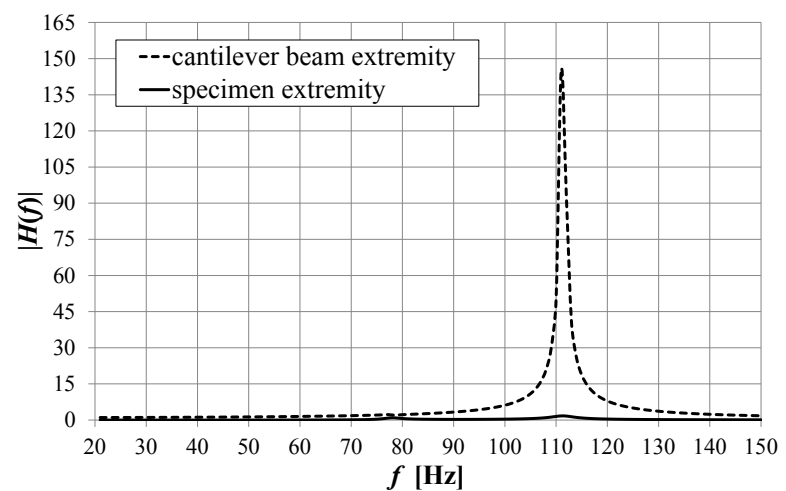

Fig. 5. Amplitude FRF $|\mathrm{H}(\mathrm{f})|$ under a horizontal acceleration.

In the second harmonic analysis, a horizontal base acceleration was applied to induce a torsional loading and a corresponding shear stress in the notched section. Fig. 5 shows (dashed line) the amplitude FRF in terms of horizontal acceleration of the cantilever extremity (point C). The trend is the same as that shown previously: it is close to unity at low frequencies and it exhibits a peak around the first torsion resonance. The solid line is the FRF in terms of vertical acceleration of specimen extremity (point B). Here too the FRF is close to zero at low frequencies and displays two peaks around the first two resonances (hardly detectable in Fig. 5), then it becomes almost zero at higher frequencies.

The trends shown in Fig. 4 and 5 point out that the bending and torsional response of the system, undergoing a vertical and horizontal base acceleration, are fully uncoupled (or at least only weakly coupled), in the frequency range far from the resonance.

The finite element analysis also allows two types of information to be obtained: i) an evaluation of the acceleration at shaker table, necessary to break the specimen; ii) an estimate of the strains at the T-clamping system, which are correlated to bending/torsion moments and to the notch stresses. It is very important to know this information, as it ensures that laboratory tests are properly executed.

Since the purpose of the testing system here presented is to carry out fatigue tests in the high-cycle regime, it is required to estimate the acceleration values that lead to specimen failure at $5 \cdot 10^{4}$ and $2 \cdot 10^{6}$ cycles (taken as lower and upper limits of life fatigue).

Constant amplitude fatigue tests were preliminary carried out to assess the S-N curve of plain material. Cylindrical smooth specimens were submitted to a tension-compression fully-reversed $(R=-1)$ loading applied by a MTS machine. Regression analysis of experimental results returned the S-N line (Fig. 6), with a reference amplitude strength $\sigma_{\mathrm{A} 0,-1}=324.1 \mathrm{MPa}$ (at $2 \cdot 10^{6}$ cycles) and an inverse slope of S-N curve $k=14.78$.

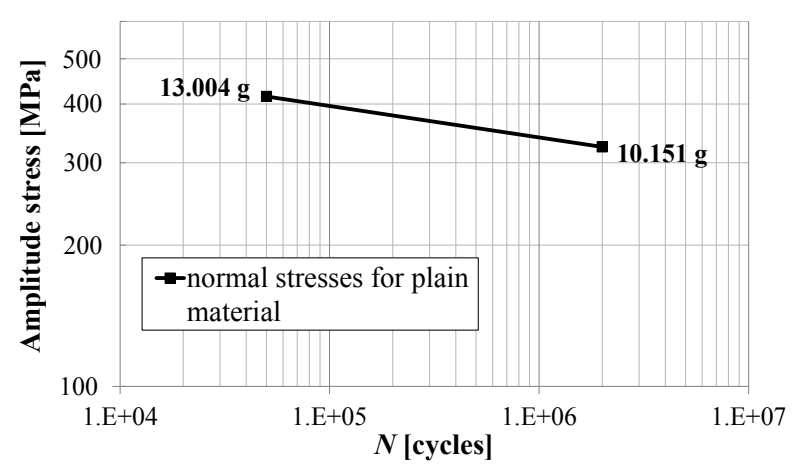

Fig. 6. S-N curve for plain material, from tension-compression tests (MTS).

A reference excitation frequency of $5 \mathrm{~Hz}$ was chosen to calculate the input acceleration that needs to be applied by the shaker in fatigue tests. Since the excitation is far from the bending resonance, the intensity of the response acceleration in every point of the testing system (also including the cantilever beam extremity) will be almost equal to the input acceleration applied by the shaker table. In Fig. 6, the numbers close to the S-N line represent the required vertical base acceleration to have for failure at $5 \cdot 10^{4}$ and $2 \cdot 10^{6}$ cycles under bending loading. 


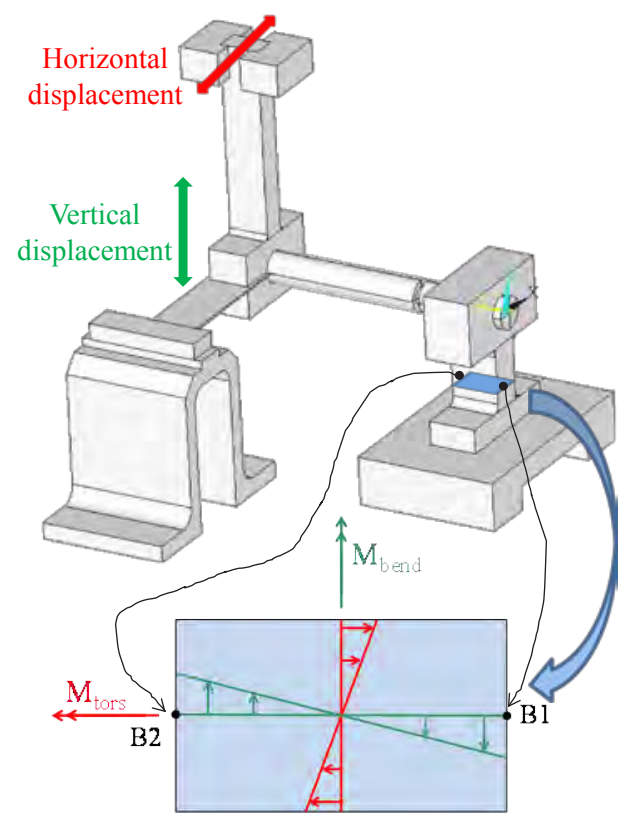

Fig. 7. Strain distribution in the T-clamp, when the specimen is subjected to bending and torsion loading.

While the graph in Fig. 6 gives information about the necessary accelerations to be applied by the shaker, before performing the laboratory tests it is also important to know the strain values in the T-clamping system. In experiments, such strains are measured and used to provide an indirect estimate of the stresses in the notched section, which being so narrow prevents any direct measure by strain gauges. In fact, the strains at the Tclamping system are directly correlated to the bending and torsion moments acting at the specimen fixed end. When the specimen bends vertically, the T-clamp will bend vertically as well. Instead, when the specimen rotates under a torsion loading, the T-clamp will bend in the plane orthogonal to the specimen axis, as sketched in Fig. 7.

Table 1. Results from finite element analysis.

\begin{tabular}{|c|c|c|c|}
\hline No. cycles & $\begin{array}{c}\text { Input } \\
\text { acceleration } \\
{[\boldsymbol{g}]}\end{array}$ & $\begin{array}{c}\text { Strain } \\
{[\boldsymbol{\mu} \boldsymbol{\varepsilon}]}\end{array}$ & $\begin{array}{c}\text { peak stress } \\
\boldsymbol{\sigma}_{\text {peak }}[\mathrm{MPa}]\end{array}$ \\
\hline $5 \cdot 10^{4}$ & 13.004 & 34.730 & 415.2 \\
\hline $2 \cdot 10^{6}$ & 10.151 & 27.115 & 324.1 \\
\hline
\end{tabular}

When the finite element model is subjected to a vertical acceleration (specimen under bending loading), the simulation returns the values of strains listed in Table 1 , calculated as the average values between points B1 and $\mathrm{B} 2$.

Table 1 summarises the estimated values of vertical base acceleration, and the corresponding strain and peak stress calculated by simulation, which are estimated to have the specimen failure at $5 \cdot 10^{4}$ and $2 \cdot 10^{6}$ cycles.

\section{Experimental fatigue tests}

Experimental measures are used for validating the finite element model and for proving that the system, when excited by a tri-axis shaker, is really capable to apply (and to control independently) a bending and/or torsion loading.

A Dongling tri-axis electrodynamic shaker (3ES-10HF-500 model) was used to perform vibration tests (Fig. $8(\mathrm{a})$ ). This brand new machine is capable to apply simultaneous accelerations along 3 orthogonal directions in space, in a frequency range from 5 to $2000 \mathrm{~Hz}$, with a maximum rated acceleration of $9.0 \mathrm{~g}$ and a maximum rated velocity of $1.2 \mathrm{~m} / \mathrm{s}$. The controller and acquisition unit is a LMS SCADAS Mobile, driven by LMS Test.Lab software.
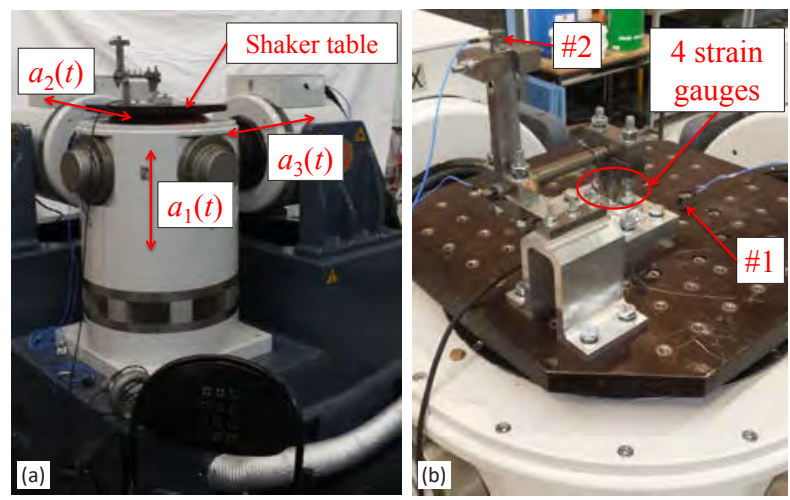

Fig. 8. (a) Dongling tri-axis electrodynamic shaker; (b) testing system prototype mounted to the shaker table.

The shaker is not capable to excite only one single or two axes (channels) at a time, keeping the others at rest. All three axes (channels) must always be activated simultaneously, which could be a limitation if tests with two, or even only one single acceleration, have to be performed. Nevertheless, this restriction can easily be overcome if the excitation level of the "secondary" channel/channels is set to a much lower level than the "primary" one. For example, to apply a vertical acceleration of $1 g$, the other two channels may be set at 0.19 .

A prototype of the new system layout, described in Section 2, is mounted to the shaker head with screws and nuts, as shown in Fig. 8(b). The measurement instruments consist of a tri-axis accelerometer (\#1) placed on shaker table in order to measure the input accelerations in closed loop with the control system, and another accelerometer (\#2) for monitoring the system response at the extremity of cantilever beam (point $\mathrm{C}$ in Fig. 2).

Four strain gauges (with half-bridge connection) have been positioned on the faces of the T-clamp (Fig. $8(\mathrm{~b})$ ), because their application on the notch $(2 \mathrm{~mm}$ of radius) was clearly not feasible.

Preliminary harmonic tests with sinusoidal acceleration were carried out with the aim to perform bending fatigue tests with constant amplitude stress. Before starting the tests, it was noticed that the acceleration values calculated by the numerical model, and required to produce failures while exciting at a frequency far below the bending resonance $(78.7 \mathrm{~Hz})$, 
were actually too higher than the threshold (9.0g) imposed by the shaker available in the laboratory.

Some amplification of the input acceleration then becomes necessary. This can be achieved by exploiting the amplification in the FRF (see Fig. 4), which occurs also at frequency below the resonance. To this purpose, a harmonic analysis was performed to evaluate with greater precision the amplitude FRF of the system under bending. In this case, three harmonic (sinusoidal) accelerations with frequency varying from 20 to $150 \mathrm{~Hz}$ (in steps of $0.05 \mathrm{~Hz}$ ) were applied to the shaker table. The acceleration $a_{1}(t)$ is along the vertical direction and has amplitude $1 g$, whereas the other two horizontal accelerations $a_{2}(t), a_{3}(t)$ have a amplitude significantly smaller, equal to $0.1 g$.

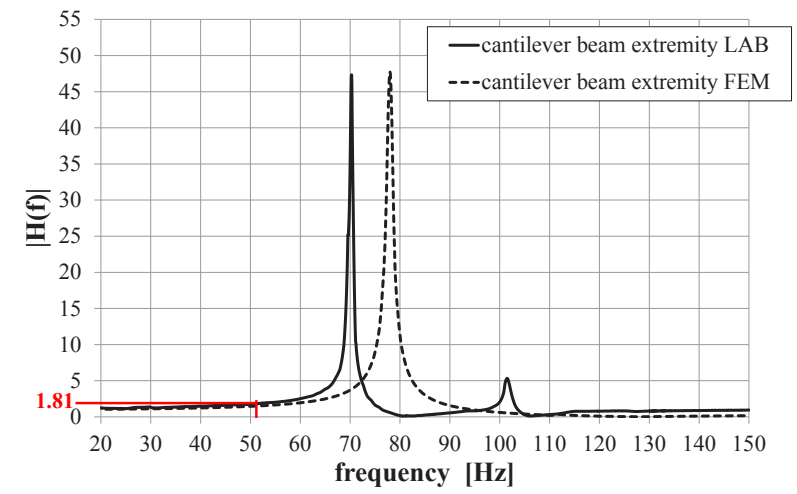

Fig. 9. Numerical (FEM) and experimental (LAB) amplitude FRF $|\mathrm{H}(\mathrm{f})|$ under vertical acceleration.

Fig. 9 shows the comparison between numerical simulation and experiments for the FRF relative to base acceleration for the output vertical acceleration at the cantilever beam extremity (accelerometer \#2, see Fig. 8(b)). The observed difference can be explained by considering that in the finite element model (Fig. 2) the assembled parts are considered to be perfectly bonded (with no joints).

Considering that the acceleration threshold allowed by the shaker is $9.0 \mathrm{~g}$ and that, by contrast, the finite element analysis estimated a maximum acceleration of $13.004 \mathrm{~g}$ to have failures around $5 \cdot 10^{4}$ cycles (see Fig. 6), the required amplification has to be about 1.44. To keep a safety margin, this requirement can be obtained by choosing a working frequency around $51 \mathrm{~Hz}$, at which the amplification is 1.81 (see Fig. 9). At this frequency, the FRF is reasonably flat and the system acceleration response, being not so sensitive to small changes in the excitation frequency, is easier to be controlled. By contrast, a more precise Input/Output control would be necessary to keep the vibrating system close to the resonance.

With these premises, fatigue tests have been carried out with a vertical harmonic base excitation of varying amplitude, whose intensity is greater than the other two horizontal accelerations ( $0.1 \mathrm{~g}$ amplitude).

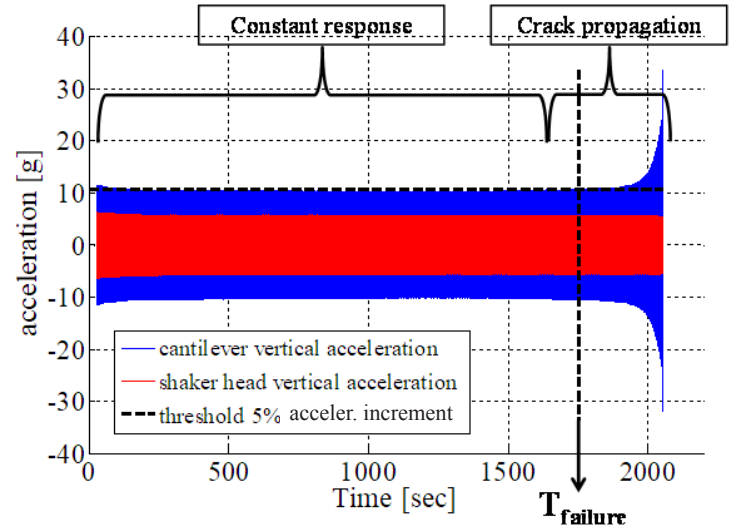

Fig. 10. Fatigue behavior under harmonic excitations.

A typical behaviour observed in this type of test is shown in Fig. 10. A first long part at constant acceleration response is followed by an increase of acceleration due to crack propagation, after which failure occurs very quickly. In the very last part of the test, significantly smaller than the first one, the crack propagation reduces the specimen stiffness, so that the system resonant frequency decreases until it reaches the frequency of the excitation. As a failure criterion, it was established to assume a $5 \%$ increment of the measured acceleration, compared to the sector in which the acceleration remains almost constant, see Fig. 10.
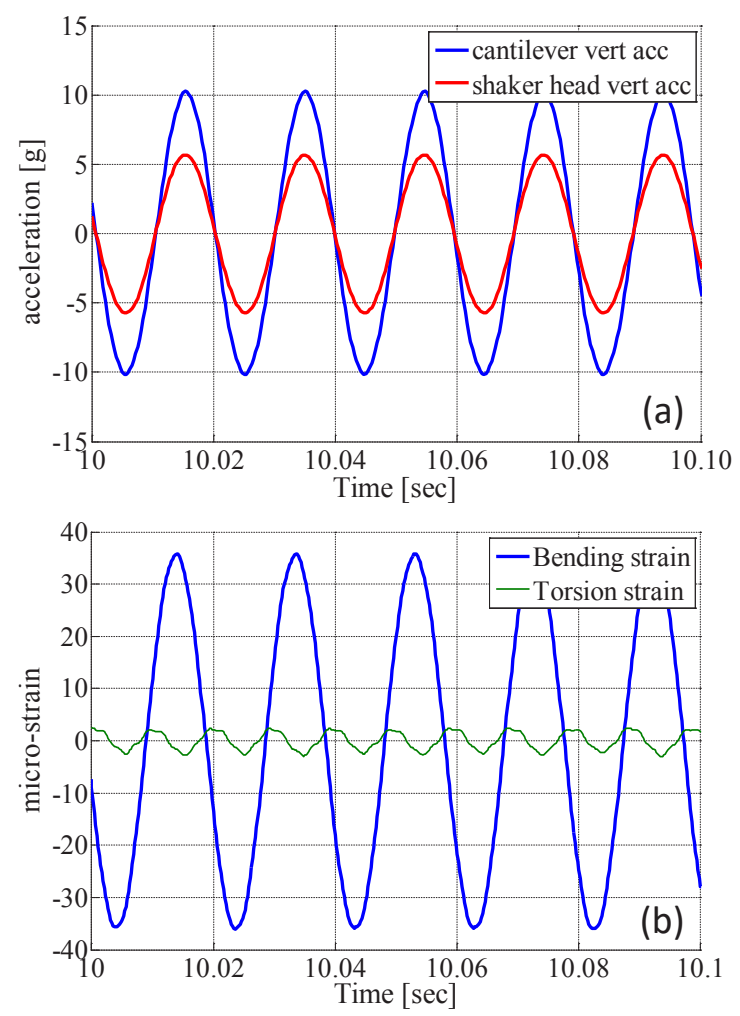

Fig. 11. (a) Acceleration measures and (b) strain measures under harmonic excitations.

Fig. 11(a) also confirms that, if the input acceleration is harmonic, the response acceleration is harmonic too. Fig. 11(b) displays, instead, the time-histories of strain measured at the T-clamp. As can be seen, the strain caused by the bending deformation is largely 
predominant to that caused by torsion loading (which yet is not zero, as the "secondary" channel for horizontal acceleration cannot be deactivated at all).

Four fatigue tests under harmonic bending have been carried out at different excitation (stress) levels. Results are summarised in Table 2, which also list information about the vertical acceleration measured with the accelerometers \#1 and \#2, and the corresponding amplitude FRF. Also the averaged strain measures at the T-clamp and the number of cycles to failure are reported. The last column lists the values of peak normal stress in the notched specimen section, which are calculated numerically using as input the measured values of strain.

Finally, Fig. 12 collects fatigue test results obtained with harmonic excitation and with tension-compression loading (MTS testing machine). A close agreement can be clearly appreciated.

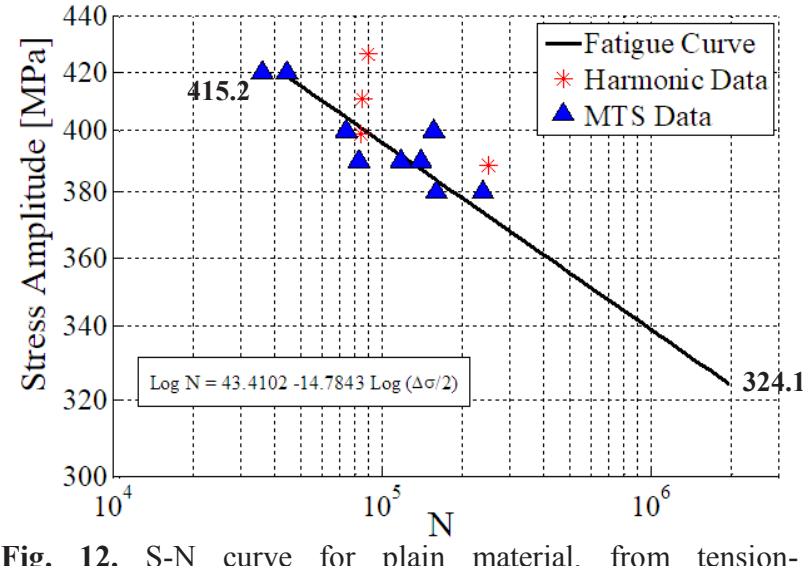

Fig. 12. S-N curve for plain material, from tensioncompression tests (MTS) and shaker tests (harmonic data)

Table 2. Results from fatigue tests under harmonic bending.

\begin{tabular}{|c|c|c|c|c|c|c|}
\hline $\begin{array}{c}\text { Test } \\
\text { No. }\end{array}$ & $\begin{array}{c}\text { Meas. input acceler. } \\
\text { (point \#1) [g] }\end{array}$ & $|\mathbf{H}(\mathbf{f})|$ & $\begin{array}{c}\text { Meas. vertical acceler. } \\
\text { (point \#2) }[\mathbf{g}]\end{array}$ & $\begin{array}{c}\text { Average strain } \\
\text { at B1/B2 }[\boldsymbol{\mu \varepsilon}]\end{array}$ & $\begin{array}{c}\text { Cycles to } \\
\text { failure }\end{array}$ & $\begin{array}{c}\text { Peak stress } \\
\boldsymbol{\sigma}_{\text {peak }}[\mathbf{M P a}]\end{array}$ \\
\hline 1 & 5.623 & 1.819 & 10.230 & 35.670 & 89295 & 426 \\
\hline 2 & 5.459 & 1.805 & 9.852 & 34.353 & 85238 & 411 \\
\hline 3 & 5.248 & 1.824 & 9.571 & 33.374 & 83622 & 399 \\
\hline 4 & 5.101 & 1.827 & 9.318 & 32.492 & 249432 & 388 \\
\hline
\end{tabular}

\section{Conclusions}

This work presented a new testing system to carry out bending-torsion vibratory tests by a tri-axis shaker. The testing system is composed of a cylindrical specimen that can be excited by three orthogonal base accelerations. An eccentric tip mass is used to produce a torsion loading under a horizontal acceleration, while thin plate attached to the specimen extremity prevents a horizontal bending when torsion is activated. A vertical acceleration, instead, produces a pure bending loading, without torsion. The phase shift between bending and torsion is thus directly proportional to the correlation among base accelerations.

A finite element model was first used to investigate the system dynamic response and to assess the local stress in the notched section of the specimen. A modal analysis allowed the first resonant frequencies in bending and torsion to be identified. A harmonic analysis was next performed by imposing a vertical and horizontal base accelerations in order to quantify the output accelerations in the specimen and the corresponding frequency response function of the system. The observed dynamic behaviour confirmed that bending and torsion deformation are truly uncoupled. The numerical results were also used to establish the excitation frequency to be used in experimental fatigue tests.
Preliminary experimental measures under a harmonic base acceleration, used to validate the results from the finite element model, confirmed the expected behaviour of the system (uncoupled bending-torsion). Four strain gauges attached to the specimen fixture were also used to monitor the strains at the clamping system, which were used to get an indirect measure of bending and torsion moments, and of the corresponding stress, in the specimen. The comparison between numerical and experimental results showed an overall fairly good correlation, thus confirming that bending and torsion loading can be controlled independently.

The works concluded by illustrating few results of constant amplitude fatigue tests obtained by a harmonic (sinusoidal) vertical base acceleration (pure bending loading). These results were in close agreement to the constant amplitude S-N curve for plain material, previously estimated by a tension-compression constant amplitude-reversed loading.

\section{References}

1. D. Benasciutti, R. Tovo, Int. J. Fatigue 27(8), 867 (2005)

2. D. Benasciutti, R. Tovo, Probab. Eng. Mech. 21(4), 287 (2006)

3. D. Benasciutti, J. Sound Vib. 333(18), 4326 (2014) 
4. D. Benasciutti, F. Sherratt, A. Cristofori, Int. J. Fatigue 91(2), 397 (2016)

5. D. Benasciutti, R. Tovo, Mat.-wiss. u. Werkstofftech. 49(3), 345 (2018)

6. T. George, Int. Journal of Fatigue 26(5), 477 (2004)

7. C. Ghielmetti, R. Ghelichi, M. Guagliano, F. Ripamonti, S. Vezzù, Procedia Engineering 10, 2892 (2011)

8. T. Łagoda, E. Macha, A. Niesłony, Fatigue Fract. Eng. Mater. Struct. 28(4), 409-420 (2005)

9. M. Česnik, J. Slavić, M. Boltežar, J. Sound Vib. 331(24), 5370 (2012)

10. N. Nguyen, M. Bacher-Höchst, C.M. Sonsino, Mat.wiss. u. Werkstofftech. 42(10), 904 (2011)

11. D. Zanellati, D. Benasciutti, R. Tovo, Proc. Struct. Integrity 8, 92 (2018)

12. W. Pilkey, D. Pilkey, R. Peterson, Peterson's stress concentration factors (John Wiley \& Sons, Hoboken, NJ, 2008) 